\title{
Pulmonary artery pseudoaneurysm secondary to metastatic angiosarcoma
}

A 70 year old woman presented to the emergency department with haemoptysis and right sided chest pain. Of significance was a prior history of angiosarcoma arising from soft tissues in the neck. A contrast enhanced helical CT scan of the thorax was performed to exclude pulmonary emboli. Multiple lung nodules were seen, some of which were cavitary. This was associated with a small left hydropneumothorax and a small right pleural effusion (fig lA). Several lung nodules were surrounded by ground glass opacity, and one of the right lower lobe lung metastases was observed to be contiguous with a subsegmental branch of the right anterior basal pulmonary artery and a pseudoaneurysm (fig 1B) which was better appreciated on the maximum intensity projection (MIP) image (fig 2). There was no history of pulmonary angiography, Swan-Ganz catheterisation, percutaneous needle biopsy of the lung, or pneumonia. The patient had no further haemoptysis and the chest pain abated on its own after a few hours so, because of her age and diffuse metastatic disease, no intervention was performed. It is presumed that the pseudoaneurysm resulted from erosion of an angiosarcoma metastasis into a subsegmental pulmonary artery resulting in haemoptysis.

Angiosarcoma lung metastases are commonly solid nodules and thin walled cysts often admixed with haemorrhage. ${ }^{1}$ The CT halo sign (lung nodule with surrounding ground glass density) has been described in angiosarcoma metastases because of haemorrhage into the surrounding lung parenchyma. Subpleural cystic lesions can give rise to pneumothorax, as illustrated in this case. However, pulmonary artery pseudoaneurysm is an unusual and previously unreported manifestation of angiosarcoma metastases. Pulmonary artery pseudoaneurysms are commonly iatrogenic or mycotic in origin. ${ }^{2}$ They have rarely been reported secondary to tumours. ${ }^{2-5}$

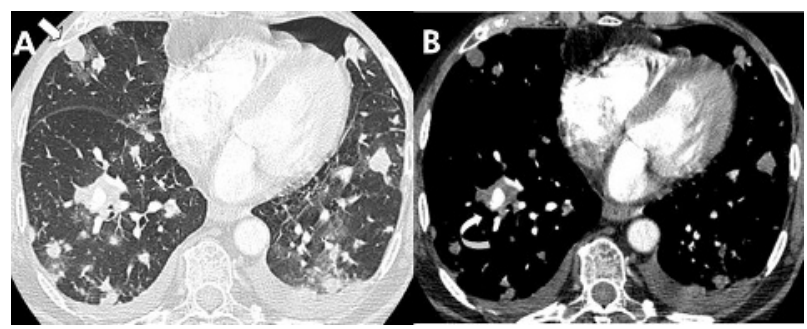

Figure 1 (A) Axial chest CT scan showing multiple lung metastases bilaterally with adjacent ground glass halo (arrow) due to haemorrhage along with a left hydropneumothorax and a small right pleural effusion. (B) Mediastinal window showing the right lower lobe metastasis eroding a subsegmental branch of the anterior basal right pulmonary artery with pseudoaneurysm formation (curved arrow).

\section{Learning points}

- Angiosarcoma metastases to the lungs can be cavitary leading to pneumothorax.

- Lung metastases are frequently haemorrhagic and may demonstrate the $\mathrm{CT}$ halo sign.

- Pulmonary artery pseudoaneurysm is an uncommon manifestation of angiosarcoma metastatic to the lungs and can be diagnosed confidently on contrast enhanced helical CT scans.

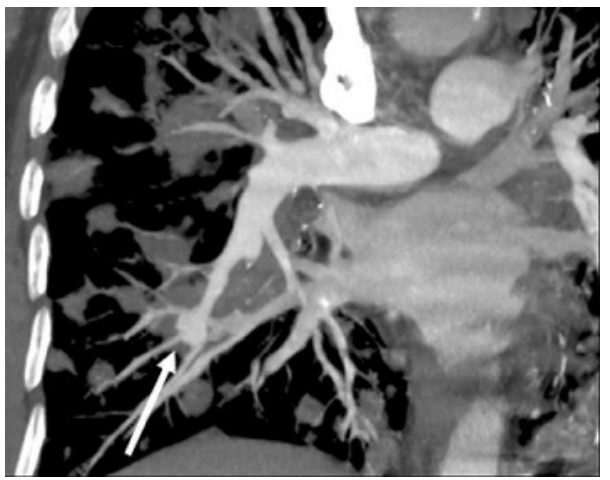

Figure 2 Coronal MIP image showing lung metastases with pseudoaneurysm of a subsegmental pulmonary artery (arrow).

P P Agarwal, C J Dennie, F R Matzinger, R A Peterson, J M Seely Department of Diagnostic Imaging, The Ottawa Hospital, Ottawa, Ontario, Canada

Correspondence to: $\operatorname{Dr}$ C J Dennie, Department of Diagnostic Imaging, The Ottawa Hospital, Civic Campus, 1053 Carling Avenue, Ottawa, Ontario K1Y 4E9, Canada; cdennie@ottawahospital.on.ca

\section{REFERENCES}

1 Tateishi U, Hasegawa T, Kusumoto $M$, et al. Metastatic angiosarcoma of the lung: spectrum of CT findings. Am J Roentgenol 2003;180:1671-4.

2 Oliver TB, Stevenson AJ, Gillespie IN. Pulmonary artery pseudoaneurysm due to bronchial carcinoma. Br J Radiol 1997;70:950-1.

3 Gomez-Jorge J, Mitchell SE. Embolization of a pulmonary artery pseudoaneurysm due to squamous cell carcinoma of the lung. J Vasc Intervent Radiol 1999;10:1127-30.

4 Ablett MJ, Elliott ST, Mitchell L. Case report: pulmonary leiomyosarcoma presenting as a pseudoaneurysm. Clin Radiol 1998;53:851-2.

5 Bean SH, Sadler DJ, Gray RR, et al. Embolization of pulmonary pseudoaneurysms secondary to metastatic synovial sarcoma of the right atrium. J Vasc Intervent Radiol 1999;10:649-52. 